\title{
Use of Complementary Health Approaches for Chronic Low-Back Pain: A Pain Research Registry-Based Study
}

\author{
John C. Licciardone, DO, MS, MBA, and Vishruti Pandya, MBBS, MHA
}

\begin{abstract}
Objectives: To measure the use of complementary health approaches (CHAs) recommended in recent clinical practice guidelines relating to low-back pain, multivariate factors associated with their use, and clinical outcomes of CHA users and nonusers.

Design: Observational cross-sectional study.

Settings/Location: The Pain Registry for Epidemiological, Clinical, and Interventional Studies and Innovation.

Subjects: A total of 568 patients with chronic low-back pain.

Interventions: Massage therapy, spinal manipulation, yoga, and acupuncture.

Outcome measures: The numerical rating scale for low-back pain intensity, Roland-Morris Disability Questionnaire for back-related disability, and the Patient-Reported Outcomes Measurement Information System with 29 items for quality-of-life deficits relating to sleep disturbance, pain interference with activities, anxiety, depression, and low energy/fatigue.

Results: The distribution of the number of different CHAs used by patients for low-back pain was as follows: $0,179(31.5 \%) ; 1,139(24.5 \%) ; 2,160(28.2 \%) ; 3,70(12.3 \%)$; and 4, $20(3.5 \%)$. The numbers of patients using the specific CHAs were as follows: massage therapy, 271 (47.7\%); spinal manipulation, 238 (41.9\%); yoga, 144 (25.4\%); and acupuncture, $96(16.9 \%)$. Opioids had been used for low-back pain by $415(73.1 \%)$ patients. Higher levels of education and higher pain self-efficacy scores were associated with greater use of any CHA, whereas increasing age and being Black were associated with lesser use of any CHA. Any CHA use was associated with lesser low-back pain intensity and lesser back-related disability. Patients who used massage therapy reported better clinical outcomes across all three dimensions. Patient pain self-efficacy also enhanced the effect of CHA use.

Conclusions: The use of CHAs relative to opioids for low-back pain was inconsistent with recommendations from recent clinical practice guidelines despite clinical benefits with CHA use in this study. More research is needed on ways to improve the uptake of CHAs recommended for low-back pain, particularly among older and Black patients.
\end{abstract}

Keywords: chronic low-back pain, complementary health approaches, nonpharmacologic therapy, physical functioning, quality of life, pain research registry

\section{Introduction}

T he Centers for Disease Control and Prevention (CDC) Guideline for Prescribing Opioids for Chronic Pain in 2016 recommended nonpharmacologic therapy as an initial preferred option for patients with chronic pain. ${ }^{1}$ This recommendation applies to all persons and is based on randomized controlled trials or observational studies. The American College of Physicians Clinical Practice Guideline in 2017 similarly recommended nonpharmacologic therapies, 
including massage therapy, spinal manipulation, yoga, and acupuncture, as first-line treatments for patients with lowback pain. ${ }^{2}$ Their recommendation is based on low- to moderate-quality evidence for each of the various nonpharmacologic therapies. Recently reported findings from the National Ambulatory Medical Care Survey demonstrate that massage therapy, spinal manipulation, herbs and nonvitamin supplements, yoga, and acupuncture were the most common complementary health approaches (CHAs) recommended by physicians in the United States. ${ }^{3}$ In light of these major clinical practice guidelines and survey findings, the authors used a pain research registry to measure the use of massage therapy, spinal manipulation, yoga, and acupuncture reported by patients with chronic low-back pain, factors associated with their use, and the clinical outcomes of CHA users and nonusers in terms of low-back pain intensity, back-related disability, and quality-of-life deficits.

\section{Materials and Methods}

\section{Study design and registry overview}

This observational cross-sectional study was conducted within the Pain Registry for Epidemiologic, Clinical, and Interventional Studies and Innovation (PRECISION Pain Research Registry) from April 2016 through November 2019. Having chronic low-back pain is a current requirement for enrollment in the registry, which recruits patients from clinics, through community organizations, and by social media. Women who are pregnant and institutionalized persons are excluded from the registry. An overview describing historical aspects of the registry has been published. ${ }^{4}$ The methods described below refer specifically to the present study.

\section{Inclusion and exclusion criteria}

Patient inclusion criteria were as follows: (1) being 21-79 years of age; (2) having English language proficiency to complete registry case report forms; and (3) having chronic low-back pain based on the diagnostic criteria established by the National Institutes of Health (NIH) Task Force on Research Standards for Chronic Low Back Pain. ${ }^{5}$ The latter require pain persistence for at least 3 months and pain frequency of at least half the days in the past 6 months. The study was approved by the University of North Texas Institutional Review Board and all patients provided written informed consent before participation.

\section{Data sources and measurement}

All patients reported if they had ever used any of the four CHAs under investigation for low-back pain at the baseline registry encounter. They also reported if they had ever received opioids for low-back pain using an item that listed over 20 generic or trade name exemplars for common opioid products used in the United States. Additional data for this study included elements of the minimum dataset recommended by the NIH Task Force on Research Standards for Chronic Low Back Pain ${ }^{5}$ to describe patient characteristics, the Pain Catastrophizing Scale (PCS), ${ }^{6}$ and the Pain Self-Efficacy Questionnaire (PSEQ). ${ }^{7}$ Scores on the latter psychological measures were trichotomized as low, moderate, or high based on the respective terciles observed within the study patients. Clinical outcomes were measured with a numerical rating scale (NRS) for average low-back pain intensity over the past 7 days, the Roland-Morris Disability Questionnaire (RMDQ) ${ }^{8}$ for back-related disability, and the Patient-Reported Outcomes Measurement Information System with 29 items (PROMIS-29) ${ }^{9}$ for quality of life. The latter was used to derive the sleep disturbance, pain interference with activities, anxiety, depression, and low energy/fatigue (SPADE) cluster score as a composite measure of quality-of-life deficits. ${ }^{10}$

\section{Statistical analysis}

The patient characteristics were summarized using the number and percentage for categorical variables and the mean and standard deviation for continuous variables. Multiple logistic regression was used to compute odds ratios (ORs) and 95\% confidence intervals (CIs) for demographic characteristics, psychological measures, and opioid use as predictors or potential confounders of the use of any of the four CHAs for low-back pain (massage therapy, spinal manipulation, yoga, or acupuncture). Similarly, ORs and 95\% CIs were computed for CHA-specific use for low-back pain. The $t$-test was used to assess differences in clinical outcomes (low-back pain intensity, back-related disability, and quality-of-life deficits) according to any CHA use and specific use of each CHA for low-back pain. Each clinical outcome was then dichotomized to further assess the relationship between any CHA use and prevention of adverse clinical outcomes. Low-back pain intensity was dichotomized as severe pain (NRS score, 7-10) versus mild-tomoderate pain (NRS score, 0-6) based on a commonly used categorization system. Back-related disability was dichotomized as high (RMDQ score, 18-24) versus low-to-moderate (RMDQ score, 0-17). Quality of life was dichotomized as poor (SPADE score, 60.0-76.4) versus good-to-fair (SPADE score, 37.7-59.9). The two latter dichotomizations were based on approximate upper tercile cut points for scores on each underlying measure. Overall, ORs and 95\% CIs were computed for any CHA use as predictors of each of the three adverse clinical outcomes. Similarly, such ORs and 95\% CIs were computed within subgroups based on demographic characteristics, psychological measures, and opioid use to identify potential effect modifiers using tests for interaction. Data were managed and analyzed with the IBM SPSS Statistics software (version 25) and all hypotheses were tested at the 0.05 level of statistical significance.

\section{Results}

A total of 568 patients with chronic low-back pain were included in the study. The mean age of patients was 53.2 years (SD, 12.5 years) and there were 401 (70.6\%) female patients (Table 1). A majority of patients had low-back pain for more than 5 years, almost one-half reported a history of depression, and almost three-fourths had used opioids for low-back pain. The distribution of the total number of different CHAs used by a patient for low-back pain was as follows: 0, 179 (31.5\%); 1, 139 (24.5\%); 2, 160 (28.2\%); 3, $70(12.3 \%)$; and 4, $20(3.5 \%)$. The numbers of patients reporting specific use of each CHA were as follows: massage therapy, 271 (47.7\%); spinal manipulation, 238 (41.9\%); yoga, 144 (25.4\%); and acupuncture, 96 (16.9\%). 
Table 1. Sociodemographic and Clinical Characteristics of the Patients $(N=568)$

\begin{tabular}{lr}
\hline Characteristic & No. (\%) \\
\hline Age (years), mean \pm SD & $53.2 \pm 12.5$ \\
Female gender & $401(70.6)$ \\
Non-White race & $173(30.5)$ \\
Hispanic ethnicity & $70(12.3)$ \\
College education or higher & $194(34.2)$ \\
Current cigarette smoker & $113(19.9)$ \\
Low-back pain duration greater than 5 years & $371(65.3)$ \\
Ever unemployed or unable to do usual work & $218(38.4)$ \\
$\quad$ for 1 month or longer due to low-back pain & \\
Ever received disability or workers' & $133(23.4)$ \\
$\quad$ compensation benefits relating to low-back & \\
$\quad$ pain & \\
Ever involved in a lawsuit or legal claim & $57(10.0)$ \\
$\quad$ relating to low-back pain & \\
Pain Catastrophizing Scale score (mean \pm SD) & $18.6 \pm 13.9$ \\
Pain Self-Efficacy Questionnaire score & $34.5 \pm 15.2$ \\
$\quad \quad$ mean \pm SD) & \\
History of depression & $273(48.1)$ \\
Ever used opioids for low-back pain & $415(73.1)$ \\
Ever had surgery for low-back pain & $81(14.3)$
\end{tabular}

Results are displayed as no. (\%) unless otherwise indicated.

${ }^{a}$ The distribution of non-White races was as follows: American Indian or Alaskan Native, 10 (1.8\%); Asian, 6 (1.1\%); Black, 154 (27.1\%); and Native Hawaiian or other Pacific Islander, 3 (0.5\%). $\mathrm{SD}$, standard deviation.

Overall, higher levels of education and higher pain selfefficacy scores were associated with greater use of any CHA for low-back pain (Table 2). Conversely, increasing age and being non-White were associated with lesser use of any CHA. It is important to note that the non-White category consisted of $154(89.0 \%)$ Black patients and that the results for this category may not reflect the experiences of other racial groups. A subgroup analysis for the use of any CHA for low-back pain found the values of OR, 0.43 ; $95 \% \mathrm{CI}$, $0.28-0.67 ; p<0.001$, for Black versus White referents. Although the factors associated with specific CHA use were generally similar to those associated with any CHA use, there were several other significant factors identified. Hispanic patients were less likely to use spinal manipulation. Higher pain catastrophizing scores were associated with greater use of spinal manipulation and yoga, whereas opioid use was associated with greater use of acupuncture.

Patients who used any CHA reported lesser low-back pain intensity (mean, 5.9; 95\% CI, 5.7-6.1) than patients who had not used any CHA (mean, 6.5; 95\% CI, 6.2-6.8) $(p=0.001)$. Users of any CHA also reported lesser back-related disability (mean, 13.7; 95\% CI, 13.1-14.2, vs. mean, 15.0; 95\% CI, 14.1-15.8) $(p=0.01)$. However, better quality-of-life outcomes were not reported by any CHA users. Patients who used massage therapy reported better clinical outcomes across all three dimensions (i.e., lesser low-back pain intensity, lesser back-related disability, and lesser quality-oflife deficits) than did nonusers (Fig. 1). Spinal manipulation was associated with lesser low-back pain intensity, whereas yoga was associated with lesser back-related disability. However, acupuncture was not associated with better clinical outcomes along any dimension.
The use of any CHA was associated with less frequent reporting of severe low-back pain intensity (OR, 0.57; 95\% CI, $0.40-0.82 ; p=0.002$ ) (Table 3 ). Pain self-efficacy was an effect modifier, with patients having higher pain selfefficacy scores less often reporting severe low-back pain intensity with any CHA use (OR, 0.29; 95\% CI, 0.14-0.59; $p<0.001$ ) ( $p$ for interaction, 0.03). Any CHA use was not associated with less frequent reporting of back-related disability or poor quality of life.

\section{Discussion}

The authors found that higher levels of education and higher pain self-efficacy scores were associated with greater use and that older age and being Black were associated with lesser use of at least one of the four CHAs recommended for low-back pain. Importantly, these findings were based on multivariate analysis that adjusted for sociodemographic characteristics, psychological measures, and opioid use. No individual CHA was used for low-back pain by a majority of patients. Furthermore, almost one-third of patients had never used any of these four CHAs for low-back pain. These findings highlight the importance of increasing the uptake of these CHAs recommended for low-back pain, particularly among older and Black patients.

The global gap between evidence and practice in the treatment of low-back pain has been recently reviewed and possible explanations for the gap proposed. ${ }^{11}$ In Australia, primary care physicians prescribed medication and ordered imaging in $65 \%$ and $24 \%$, respectively, of new low-back pain episodes. ${ }^{12}$ In South Africa, $90 \%$ of patients seeking primary health care for low-back pain received pain medication, whereas less than $10 \%$ and $3 \%$, respectively, received spinal manipulation or acupuncture. ${ }^{13}$ Potential reasons cited for such gaps between evidence and practice may be a propensity for opioids or surgery in economically developed nations and a lack of resources to provide $\mathrm{CHAs}$ in economically underdeveloped nations. ${ }^{11}$ Implementation science is emerging as a field of research to help improve the translation of research findings into clinical practice. Although implementation science in the low-back pain field is scarce, there appears to be marginal evidence to support audit and feedback as being effective in changing practice behaviors toward best practice recommendations. ${ }^{14}$ However, such studies have not typically assessed CHA use. Approaches to CHA implementation may involve providing additional training to existing primary care providers or integrating $\mathrm{CHA}$ providers into primary care clinics to improve access to treatments. ${ }^{15}$

The recently reported findings from the National Ambulatory Medical Care Survey ${ }^{3}$ indicate that physicians are predisposed to recommending such CHAs as massage therapy, spinal manipulation, yoga, and acupuncture for low-back pain. However, insurance coverage and out-ofpocket costs for CHAs are important factors that may affect uptake and the authors did not have the relevant data to include these in their analyses. Research has now been reported on the coverage of massage therapy, spinal manipulation (i.e., chiropractic care), and acupuncture for low-back pain by 45 commercial, Medicaid, and Medicare Advantage health plans following dissemination of the CDC Guideline for Prescribing Opioids for Chronic Pain. ${ }^{16}$ The study found that most plans 


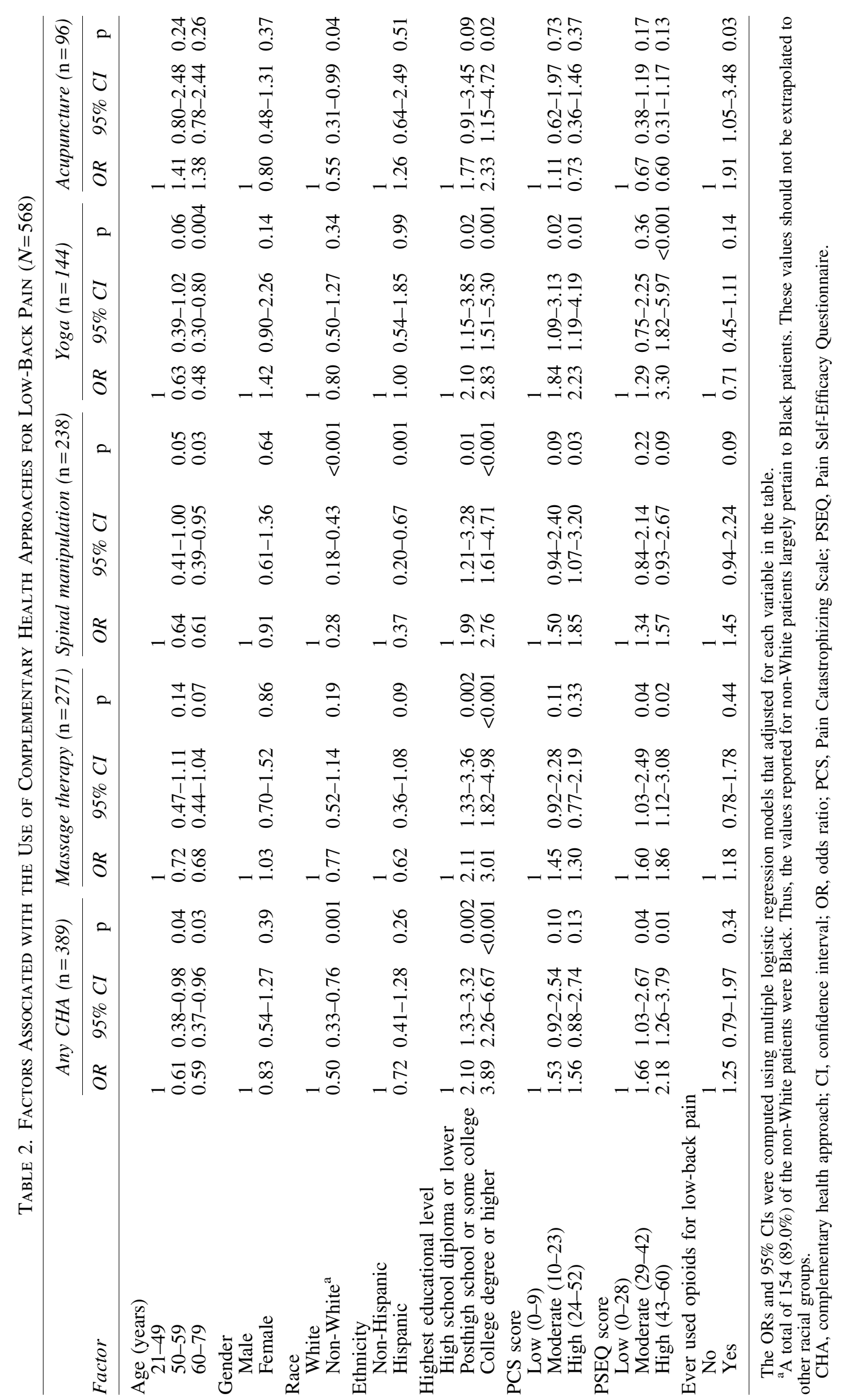



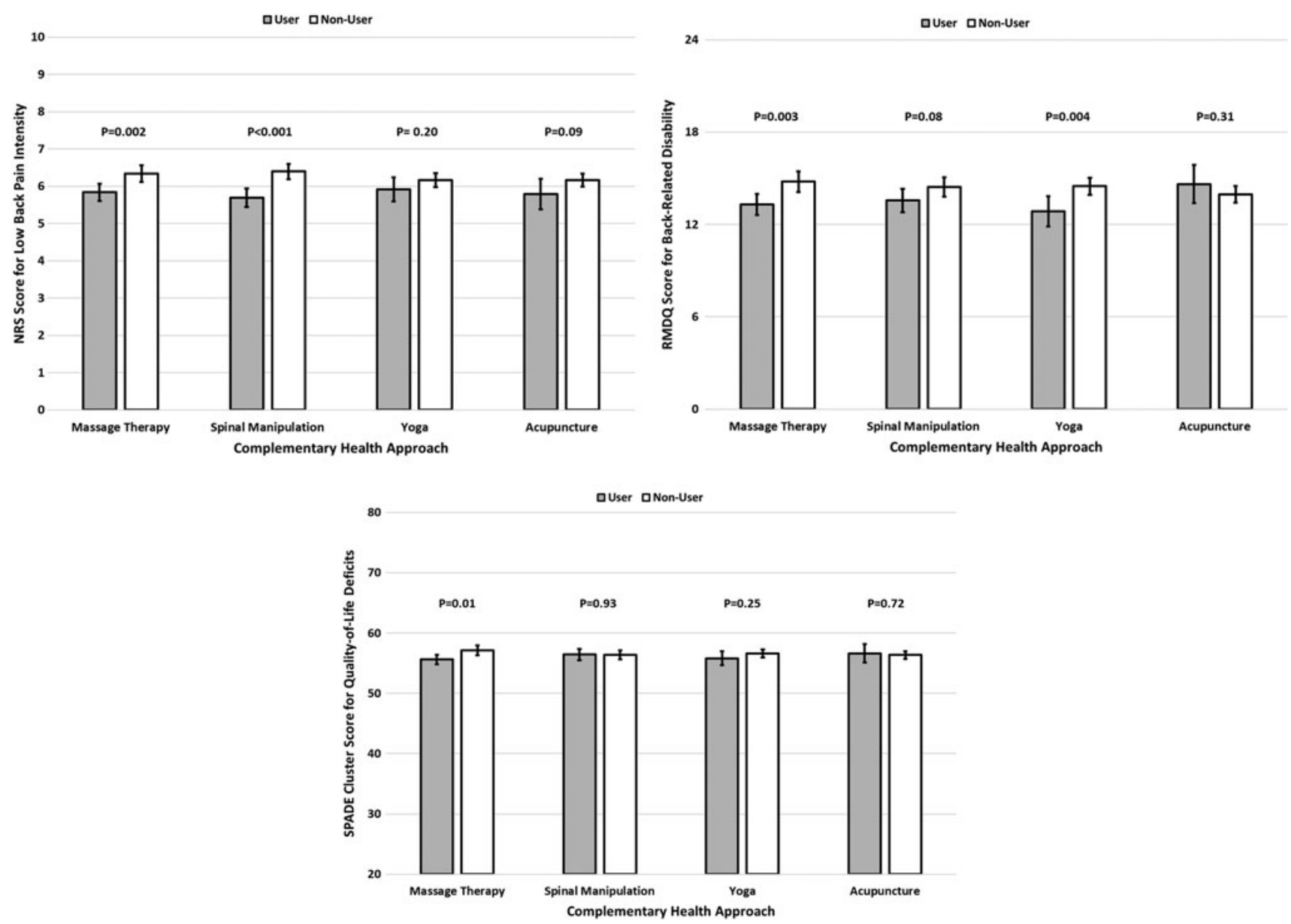

FIG. 1. Clinical outcomes according to reported use of complementary health approaches. The error bars represent $95 \%$ CIs. Higher scores represent worse clinical outcomes on each measure. CI, confidence interval; NRS, numerical rating scale; RMDQ, Roland-Morris Disability Questionnaire; SPADE, sleep disturbance, pain interference with activities, anxiety, depression, and low energy/fatigue.

covered chiropractic care, but not massage therapy or acupuncture. Thus, patients without health insurance or with limited disposable income would likely have greater difficulty in accessing any of the four CHAs studied. More research is needed on the relative strengths of patient education and selfefficacy in seeking CHAs for low-back pain versus the economic barriers in acquiring such care.

The use of any CHA was associated with lesser low-back pain intensity and lesser back-related disability. Massage therapy was the only CHA that was associated with better clinical outcomes across all three dimensions. High pain self-efficacy emerged as an important factor in this study on two grounds. First, higher pain self-efficacy scores were associated with greater use of any CHA for low-back pain. Second, when patients with high pain self-efficacy used any CHA for low-back pain, they less often reported severe lowback pain intensity than patients with low-to-moderate pain self-efficacy who used any CHA.

The use of an observational cross-sectional design to conduct this study has both limitations and advantages that should be carefully considered and weighed to help interpret the findings. There were potential limitations in using cross- sectional data that were exclusively self-reported by patients at their baseline registry encounter. Aside from being uncorroborated through medical records, such data may not have adequately captured the recency and intensity of CHA use by patients. It is possible that CHAs used in the past, or for only a limited time, may not have provided an enduring treatment effect that was discernable at the baseline encounter. This phenomenon may have attenuated the differences in clinical outcomes between CHA users and nonusers. Unlike the case with randomized controlled trials, an association between CHA use and better clinical outcomes in this cross-sectional study cannot be assumed to necessarily reflect causality because of the unclear temporal relationship between the variables. Nevertheless, randomized controlled trials may have shortcomings with regard to patient selection, protocol relevance, and the generalizability of their findings. Although the authors' research registry may not necessarily reflect the national experience, it is more likely to assess CHAs in a pragmatic manner that better reflects patient use and adherence in the real world. Consequently, the findings may be more generalizable than those derived from randomized controlled trials. 


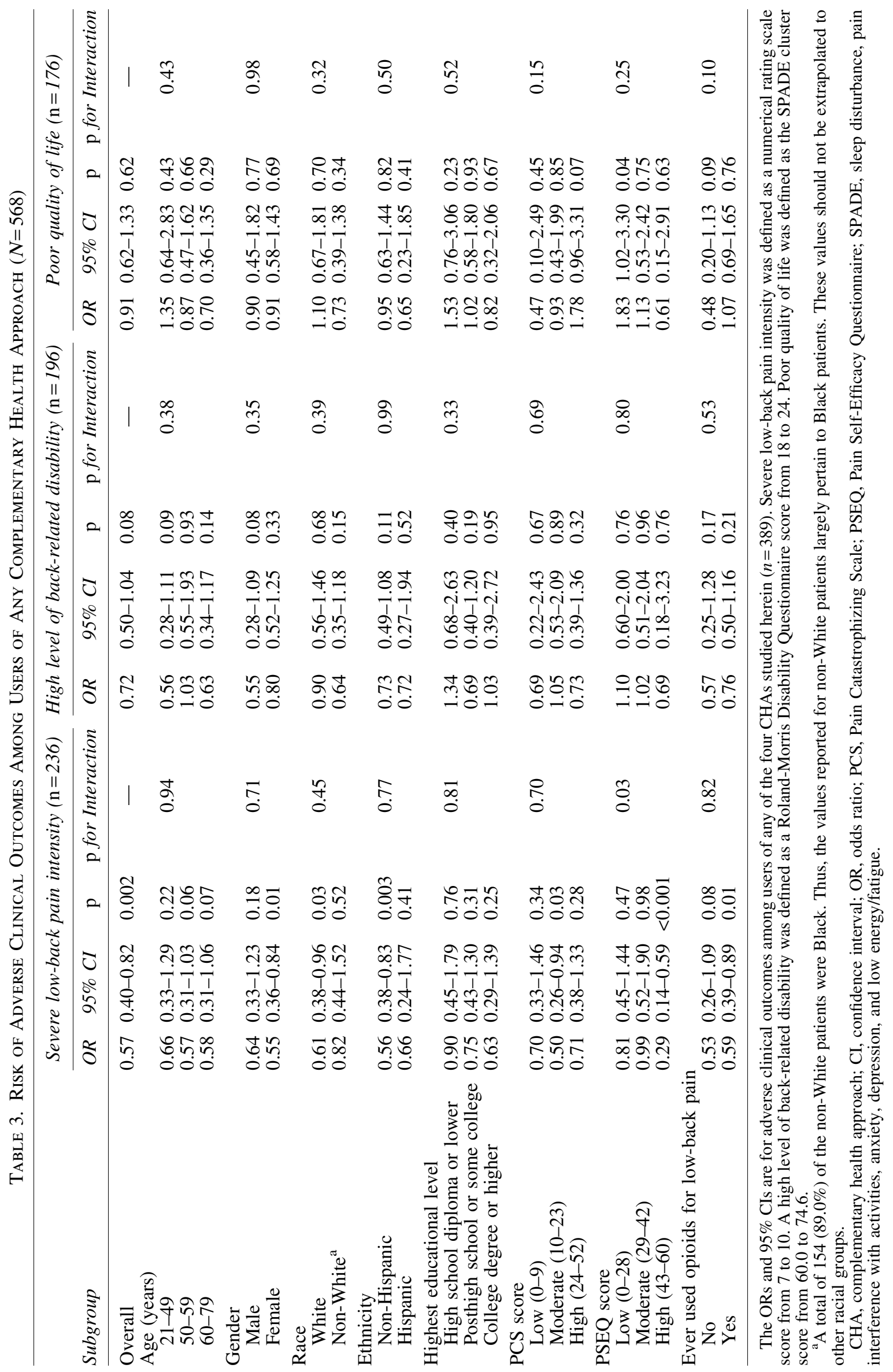




\section{Conclusions}

In summary, despite their recommended use as first-line therapies for pain in recent clinical practice guidelines, ${ }^{1,2}$ none of the four CHAs studied herein were used for lowback pain by a majority of patients and almost one-third of patients had never used any of these CHAs for low-back pain. Contrary to these guidelines, more patients reported having used opioids for low-back pain than all four CHAs combined. More research is needed on ways to improve the uptake of CHAs recommended for low-back pain, particularly among older and Black patients. Potential paths may involve greater efforts to educate patients on the benefits of CHAs for low-back pain and to facilitate their use through implementation science. However, the impact of economic barriers to accessing CHAs must also be addressed. Patients who used any $\mathrm{CHA}$ recommended for low-back pain experienced better clinical outcomes involving lesser low-back pain intensity and lesser backrelated disability.

\section{Acknowledgments}

The authors wish to thank the research registry staff and patients for their contributions to the study.

\section{Author Disclosure Statement}

No competing financial interests exist.

\section{Funding Information}

This study was supported, in part, by the Osteopathic Heritage Foundation. This sponsor did not have a role in the design of the registry; the collection, analysis, or interpretation of data; or the writing or approval of the manuscript.

\section{References}

1. Dowell D, Haegerich TM, Chou R. CDC Guideline for Prescribing Opioids for Chronic Pain-United States, 2016. MMWR Recomm Rep 2016;65 (No. RR-1):1-49.

2. Qaseem A, Wilt TJ, McLean RM, Forciea MA; Clinical Guidelines Committee of the American College of Physicians. Noninvasive treatments for acute, subacute, and chronic low back pain: A clinical practice guideline from the American College of Physicians. Ann Intern Med 2017; 166:514-530.

3. Stussman BJ, Nahin RR, Barnes PM, Ward BW. U.S. Physician recommendations to their patients about the use of complementary health approaches. J Altern Complement Med 2020;26:25-33.
4. Licciardone JC, Gatchel RJ, Phillips N, Aryal S. The Pain Registry for Epidemiological, Clinical, and Interventional Studies and Innovation (PRECISION): Registry overview and protocol for a propensity score-matched study of opioid prescribing in patients with low back pain. J Pain Res 2018; 11:1751-1760.

5. Deyo RA, Dworkin SF, Amtmann D, et al. Report of the NIH Task Force on Research Standards for chronic low back pain. J Pain 2014;15:569-585.

6. Sullivan MJ. The Pain Catastrophizing Scale: User Manual. Montreal: McGill University, 2009.

7. Nicholas MK. The Pain Self-Efficacy Questionnaire: Taking pain into account. Eur J Pain 2007;11:153-163.

8. Roland M, Morris R. A study of the natural history of back pain. Part I: Development of a reliable and sensitive measure of disability in low-back pain. Spine 1983;8:141-144.

9. PROMIS Adult Profile Instruments. Evanston, IL: Northwestern University, 2015.

10. Davis LL, Kroenke K, Monahan P, et al. The SPADE symptom cluster in primary care patients with chronic pain. Clin J Pain 2016;32:388-393.

11. Foster NE, Anema JR, Cherkin D, et al. Prevention and treatment of low back pain: Evidence, challenges, and promising directions. Lancet 2018;391:2368-2383.

12. Michaleff ZA, Harrison $\mathrm{C}$, Britt $\mathrm{H}$, et al. Ten-year survey reveals differences in GP management of neck and back pain. Eur Spine J 2012;21:1283-1289.

13. Major-Helsloot ME, Crous LC, Grimmer-Somers K, Louw QA. Management of LBP at primary care level in South Africa: Up to standards? Afr Health Sci 2014;14:698-706.

14. Hodder RK, Wolfenden L, Kamper SJ, et al. Developing implementation science to improve the translation of research to address low back pain: A critical review. Best Pract Res Clin Rheumatol 2016;30:1050-1073.

15. Gatchel RJ, Reuben DB, Dagenais S, et al. Research agenda for the prevention of pain and its impact: Report of the Work Group on the Prevention of Acute and Chronic Pain of the Federal Pain Research Strategy. J Pain 2018;19:837-851.

16. Heyward J, Jones CM, Compton WM, et al. Coverage of nonpharmacologic treatments for low back pain among US public and private insurers. JAMA Netw Open 2018;1:e183044.

Address correspondence to: John C. Licciardone, DO, MS, MBA Department of Family Medicine

University of North Texas Health Science Center 3500 Camp Bowie Boulevard Fort Worth, TX 76107

USA

E-mail: john.licciardone@unthsc.edu 